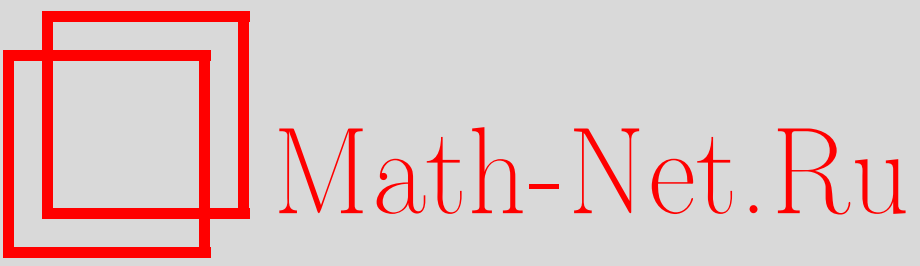

С. В. Конягин, Н. Г. Мощевитин, Представление рациональных чисел конечными цепными дробями, УМН, 1996, том 51, выпуск 4, 159-160

DOI: https://doi.org/10.4213/rm1003

Использование Общероссийского математического портала Math-Net.Ru подразумевает, что вы прочитали и согласны с пользовательским соглашением

http://www.mathnet.ru/rus/agreement

Параметры загрузки:

IP : 3.85 .5 .30

26 апреля 2023 г., 09:35:42 


\title{
ПРЕДСТАВЛЕНИЕ РАЦИОНАЛЬНЫХ ЧИСЕЛ КОНЕЧНЫМИ ЦЕПНЫМИ ДРОБЯМИ
}

\author{
С. В. Конягин, Н. Г. Мошевитин
}

Пусть $A, k, q$-натуральные числа, $q>1,1 \leqslant A<q,(A, q)=1, \varphi(q)=\#\{A\}$. Через $N(k, q)$ мы будем обозначать количество чисел $A$ таких, что $1 \leqslant A<q,(A, q)=1$ и что все неполные частные разложения в цепную дробь

$$
\frac{A}{q}=\left[0 ; B_{1}, \ldots, B_{n(A)}\right]
$$

ограничены величиной $k: B_{i} \leqslant k, i=1, \ldots, n(A)$. Известно [1], [2], что если $k>\gamma \log q$ с достаточно большой константой $\gamma$, то $N(k, q) \geqslant 1$. (Более того, для большинства чисел $A, 1 \leqslant A<q$, неполные частные ограничены величиной $O(\log q)$.) Не доказанная до сих пор гипотеза предполагает, что $N(6, q) \geqslant 1 \forall q$. В настоящей заметке мы доказываем, что чисел с ограниченными неполными частными не может быть слишком много.

Теорема 1. Для любой постоянной $\gamma<1$ и для любого $k \geqslant k(\gamma)$ имеет место оценка

$$
N(k, q) \ll \varphi(q) q^{-\frac{\gamma}{k \log k}} .
$$

Почти арифметической прогрессией (п.а.п.) назовем множество натуральных чисел $A_{1}<A_{2}<\cdots<A_{d^{*}}$, удовлетворяющее условию

$$
\exists w>0: w \leqslant A_{j+1}-A_{j} \leqslant 3 w \forall j=1, \ldots, d^{*}-1 .
$$

Через $d^{*}(k)$ обозначим такое минимальное $d^{*}$, что для любой п.а.п. $\left\{A_{1}, A_{2}, \ldots, A_{d^{*}}\right\}$ и любого $q>A_{d^{*}}$ среди разложений в конечную цепную дробь $A_{i} / q=\left[B_{i, 1}, \ldots, B_{i, n(i)}\right]$ найдется такое неполное частное $B_{i, \mu}$, для которого $B_{i, \mu}>k$. Независимо от теоремы 1 , может быть доказана

Теорема 2. Пусть $q$ - простое число. Для любой постоянной $\gamma<1$, для любого $k u$ $d=2 d^{*}(k)$ выполняется

$$
N(k, q) \ll q^{1-\frac{\gamma}{d \log d}} .
$$

Аналогично теореме из работы [3] доказывается, что $2 d^{*}(k) \ll k^{3}$, откуда и из теоремы 2 при $k>1$ вытекает более слабая, чем в теореме 1 , оценка вида $N(k, q) \ll q^{1-c /\left(k^{3} \log k\right)}$.

ДоКАЗАТЕЛЬСТво тЕОРЕмы 1 . Фиксируя $\gamma$, мы считаем, что $k$ достаточно велико и $q>k$. Положим $l=k+2$. Мы будем пользоваться тем, что если неполные частные в разложении числа $A / q, 1 \leqslant A<q,(A, q)=1$, не превосходят $k$, то при любых целых $a, b$, $0<b<q$, справедливо неравенство $|A / q-a / b|>1 /\left(l b^{2}\right)$. Пусть $n$ - натуральное число и $H_{n}=\left\{a / b \mid a, b \in N_{0}, 0 \leqslant a \leqslant b \leqslant n,(a, b)=1\right\}-$ ряд Фарея порядка $n$. Мы будем писать $H_{n}=\left\{h_{\nu}(n)\right\}(\nu=0, \ldots, \Phi(n))$ и считать, что числа $h_{\nu}(n)$ упорядочены по возрастанию:

$$
0=h_{0}(n)<\cdots<h_{\Phi(n)}(n)=1 .
$$

Пусть $l \in N$. Для каждой точки $x=a / b=h_{\nu}((l+1) b),(a, b)=1$, положим

если $x \neq 1$, и

$$
I^{+}(x)=\left[x, h_{\nu+1}((l+1) b)\right] \text {, }
$$

$$
I^{-}(x)=\left(h_{\nu-1}((l+1) b), x\right]
$$

если $x \neq 0$. Если $h_{\nu+1}((l+1) b)=c / d,(c, d)=1$, то мы имеем $b+d>(l+1) b$, т.е. $l b<d \leqslant(l+1) b$, откуда

и, аналогично,

$$
\frac{1}{(l+1) b^{2}} \leqslant\left|I^{+} \frac{a}{b}\right|<\frac{1}{l b^{2}}
$$

$$
\frac{1}{(l+1) b^{2}} \leqslant\left|I^{-} \frac{a}{b}\right|<\frac{1}{l b^{2}} \text {. }
$$

Работа выполнена при финансовой поддержке Российского фонда фундаментальных исследований (грант № 96-01-00378). 
Для $j \in Z_{+}$определим $n_{j}=(l+1)^{j}$ и

$$
E_{j+1}=[0,1] \backslash \bigcup_{x \in H_{n_{j}} \backslash\{1\}} I^{+}(x) \backslash \bigcup_{x \in H_{n_{j}} \backslash\{0\}} I^{-}(x) .
$$

Заметим, для каждого элемента $y \notin E_{j+1}$ найдется элемент $x=a / b \in H_{n_{j}}$ такой, что $|x-y|<1 /\left(l b^{2}\right)$. Положим также $E_{0}=[0,1]$.

Покажем, что

$$
\left|E_{j+1}\right| \leqslant \frac{l-1}{l+1}\left|E_{j}\right|
$$

Каждое множество $E_{j}$ по построению есть объединение отрезков вида $\left[h_{\nu}\left(n_{j}\right), h_{\nu+1}\left(n_{j}\right)\right]=$ $[a / b, c / d]$. Мы имеем

откуда

$$
E_{j+1} \cap[a / b, c / d] \subset[a / b, c / d] \backslash I^{+}(a / b) \backslash I^{-}(c / d),
$$

$$
\begin{aligned}
\left|E_{j+1} \cap[a / b, c / d]\right| & \leqslant \max \left(0,|[a / b, c / d]|-\left|I^{+}(a / b)\right|-\left|I^{-}(c / d)\right|\right) \\
& \leqslant \max \left(0,1 /(b d)-1 /\left((l+1) b^{2}\right)-1 /\left((l+1) d^{2}\right)\right) \leqslant \frac{l-1}{l+1}|[a / b, c / d]|,
\end{aligned}
$$

и (1) доказано.

Пусть $\varepsilon=(1-\gamma) / 2$ и $\mu=[(1-\varepsilon) \log q /(2 \ln (l+1))]$. В силу (1)

$$
\left|E_{\mu}\right| \leqslant\left(\frac{l-1}{l+1}\right)^{\mu}<q^{-\frac{\gamma}{k \log k}} .
$$

Множество $E_{\mu}$ есть объединение отрезков вида $\left[h_{\nu}\left(n_{\mu}\right), h_{\nu+1}\left(n_{\mu}\right)\right]=[x, y]$. Так как $n_{\mu} \leqslant q^{(1-\varepsilon) / 2}$, то длина каждого из этих отрезков не меньше $1 / q^{1-\varepsilon}>\tau(q) / \varphi(q)$, где $\tau(q)$ - число делителей $q$. По формуле включений и исключений

$$
\#\{A \in N:(A, q)=1(A / q) \in[x, y]\}<\varphi(q)(y-x)+\tau(q)
$$

$$
<\varphi(q)(y-x)+\varphi(q) / q^{1-\varepsilon} \leqslant 2 \varphi(q)(y-x) .
$$

Суммируя по всем отрезкам $[x, y]$ и учитывая $(2)$, получаем

Теорема 1 доказана.

$$
\#\left\{A \in N:(A / q) \in E_{\mu}\right\}<2 \varphi(q) q^{-\frac{\gamma}{k \log k}} .
$$

СХемА ДОКАЗАТЕЛЬСТВА ТЕОРЕМЫ 2. Введем параметры

$$
\begin{gathered}
t=q^{1-\gamma /(d \log d)}, \quad \nu=\left[2+d \log \frac{q}{t}\right] ; \\
q_{j}=q / d^{j}, \quad t_{j}=t /(d-1)^{j} ; \quad j=0, \ldots, \nu .
\end{gathered}
$$

Мы полагаем, что $d \leqslant \log q$. При достаточно болшшом $q>q_{0}\left(q_{0}\right.$ - некоторая эффективная постоянная, зависящая только от $\gamma$ ) мы имеем $q_{\nu}=q / d^{\nu}>d^{\tau}, t_{\nu} /\left(q_{\nu}+1\right)>1$. Из этих неравенств, применяя “жадный алгоритм", нетрудно доказать следующее утверждение.

Лемма. Пусть $\mathfrak{A} \subset\{1, \ldots, q-1\}$ - произвольное подмножество такое, что \# $\mathfrak{A} \geqslant t$. Тогда найдутся $j$ из интервала $1 \leqslant j \leqslant \nu$ и набор д интервалов

$$
\Delta_{1}^{(j)}=\left[q_{j} m ; q_{j}(m+1)\right), \ldots, \Delta_{d}^{(j)}=\left[q_{j}(m+d-1) ; q_{j}(m+d)\right)
$$

длины $q_{j}$ такие, что $\Delta_{i}^{(j)} \cap \mathfrak{A} \neq \varnothing \quad \forall i=1, \ldots, d$.

СлеДСТвИЕ леммы. Пусть $d^{*}=d / 2, \mathfrak{A} \subset\{1, \ldots, q-1\}-$ произвольное подмножество такое, что $\# \mathfrak{A} \geqslant t$. Тогда найдется п.a.n. $\mathfrak{B}=\left\{A_{1}, \ldots, A_{d^{*}}\right\} \subset \mathfrak{A}$.

Теорема 2 немедленно вытекает из следствия леммы, ибо $d^{*}=d^{*}(k)$.

\section{СПИСОК ЛИТЕРАТУРЫ}

[1] Коробов Н. M. // УМН. 1967. Т. 22. № 3. C. 83-118. [2] Cusick T. W. // Math. Comp. 1993. V. 61. № 203. Р. 171-176. [3] Мощевитин Н. Г. // Матем. записки. 1996. Т. 2. С. 99-109. 